\title{
Correction: Alterations in Wnt- and/or STAT3 signaling pathways and the immune microenvironment during metastatic progression
}

\author{
S. J. $\mathrm{Kim}^{1}$ · S. Garcia-Recio ${ }^{2}$ C. J. Creighton $\mathbb{(}^{3} \cdot$ C. M. Perou $\mathbb{1}^{2} \cdot$ J. M. Rosen $\mathbb{1}^{1}$
}

Published online: 26 September 2019

(c) The Author(s), under exclusive licence to Springer Nature Limited 2019

\section{Correction: Oncogene}

https://doi.org/10.1038/s41388-019-0852-0

The original version of this Article incorrectly matched the supplementary files with their titles. This has been corrected. The following information has also been added to the Methods section.

\section{Accession numbers and data sharing}

All RNAseq data newly generated here have been deposited into the Gene Expression Omnibus (GEO) under the accession number (GSE132031).

The original article can be found online at https://doi.org/10.1038/ s41388-019-0852-0.

\section{J. M. Rosen}

jrosen@bcm.edu

1 Department of Molecular and Cellular Biology, Baylor College of Medicine, Houston, TX, USA

2 Department Genetics and Lineberger Comprehensive Cancer Center, The University of North Carolina at Chapel Hill, Chapel Hill, NC, USA

3 Department of Medicine and Dan L. Duncan Comprehensive Cancer Center, Baylor College of Medicine, Houston, TX, USA 